REVIEW SERIES

\title{
Cough $\cdot$ 2: Chronic cough in children
}

\author{
J C de Jongste, M D Shields
}

Thorax 2003;58:998-1003

Chronic cough is a common problem in childhood. Viral infections are the most prevalent cause, but other rarer disorders should be excluded whenever cough appears unusually severe and/or frequent, and when there is evidence of failure to thrive and growth retardation. The younger the child, the more the need to exclude underlying disease at an early stage. Passive smoking is an important contributor to chronic cough in children. Chronic productive cough with purulent sputum is always reason for concern in children and is not common as a symptom of asthma. More or less specific diagnoses in children include cystic fibrosis, aspirated foreign body, congenital anatomic abnormalities and primary ciliary dyskinesia.

See end of article for authors' affiliations ......................

Correspondence to: Professor dr J C de Jongste, Department of Pediatrics, Sophia Children's Hospital, Erasmus MC, P O Box 2060, 3000 CB Rotterdam, The Netherlands: i.c.dejongste@ erasmusmc.nl
C hronic cough-defined as daily cough for more than 3-4 weeks - is one of the most common symptoms in childhood. While most children with cough do not have a serious lung condition, coughing can be troublesome and difficult to treat. Cough is common in asthmatic children. This overview discusses the possible diagnoses in children with troublesome coughing who otherwise appear healthy.

There are a number of differential diagnoses to consider (table 1). While "cough" is a precise symptom, its severity is often not objectively reported. Studies using overnight audio recordings have shown that parental reporting of cough does not correlate well with the frequency, duration, or intensity of the actual cough. ${ }^{1-3}$ It would appear in many cases that the reported "severity" of coughing in a child is related to how it affects the parents or teachers. A study in which cough was measured objectively found that healthy children of mean age 10 years have, on average, 10 cough episodes (range up to 34) per 24 hours, mostly during the daytime. ${ }^{4}$ This number increases during respiratory infections, of which 5-8 may occur per year in healthy children, with a duration of 7-9 days. This will cause additional coughing during another 50 days per year. ${ }^{5}$ It is likely that younger children will have more infections and, hence, even more coughing, but objective data are scarce. Questionnaire data which rely on the subjective impression of parents suggest that up to $10 \%$ of preschool and early school aged children have chronic cough without wheeze at some time. ${ }^{6-8}$ Cough without wheeze was associated with environmental factors including dampness in the home and air pollution, and is strongly related to socioeconomic status. Parental smoking is associated with an increased prevalence of chronic cough, amounting to $50 \%$ in children below 11 years of age with two smoking parents. ${ }^{9}$

Luyt reported that as many as $22 \%$ of children of preschool age reported chronic cough without colds. ${ }^{10}$ When re-examined several years later, this cough had persisted in more than one third. Children with chronic cough were not more likely to develop asthma and atopy than noncoughers, and had similar lung function and airway responsiveness. A problem with these follow up data is selection bias due to selective loss to follow up in asymptomatic children. ${ }^{11}$ It seems that coughing children have similar airway responsiveness to control children but may have a temporarily increased cough receptor sensitivity (CRS) when challenged with capsaicin. This heightened CRS (without increased airways hypersensitivity) returned to normal when the cough had resolved. A smaller group of children did not have heightened CRS but had increased airway responsiveness which also returned to normal when the cough resolved. ${ }^{12}$ Interestingly, in another study Chang et al were able to show that children with isolated cough associated with airways hypersensitivity did not respond to salbutamol and beclomethasone. This suggests that the presence of airways hypersensitivity will not be helpful in defining which coughing children are likely to benefit from antiasthma treatment. ${ }^{13}$

Chronic cough is, to some extent, normal in childhood and has a favourable prognosis with improvement being the rule in the majority. If chronic cough is unusually frequent or severe, a number of underlying disorders may be present. We classify the children into the following two groups (table 1):

- chronic isolated (no wheezing) non-specific cough in an otherwise seemingly healthy child where asthma could be in the differential diagnosis; and

- children with chronic cough in which there is a serious underlying lung condition.

\section{CHRONIC ISOLATED (NO WHEEZING) NON-SPECIFIC COUGH IN AN OTHERWISE SEEMINGLY HEALTHY CHILD WHERE ASTHMA COULD BE IN THE DIFFERENTIAL DIAGNOSIS \\ Infections}

Simple recurrent viral bronchitis is very common and is classically manifest by bouts of coughing associated with bronchitis occurring concomitantly with viral upper respiratory tract infections. These respiratory viral infections tend to occur more frequently in winter. The infections 


\begin{tabular}{|c|c|}
\hline $\begin{array}{l}\text { Isolated cough: otherwise } \\
\text { healthy child }\end{array}$ & $\begin{array}{l}\text { Isolated cough : significant underlying } \\
\text { cause }\end{array}$ \\
\hline $\begin{array}{l}\text { Recurrent viral bronchitis } \\
\text { Post-infectious cough } \\
\text { Pertussis-like illness } \\
\text { Cough variant asthma } \\
\text { Postnasal drip } \\
\text { Psychogenic cough } \\
\text { Habit ("tic" like) } \\
\text { Bizarre honking cough } \\
\text { Gastro-oesophageal reflux }\end{array}$ & $\begin{array}{l}\text { Chronic suppurative lung disease } \\
\text { - Cystic fibrosis } \\
\text { - Immune deficiencies } \\
\text { - Primary ciliary dyskinesia } \\
\text { - Recurrent pulmonary aspiration } \\
\text { - Chronic inhaled foreign body } \\
\text { Airways lesion } \\
\text { - Compression, e.g. tuberculous gland } \\
\text { - Malacia, often with viral infection, } \\
\text { e.g. TOF cough }\end{array}$ \\
\hline
\end{tabular}

may occur "back to back" and give the impression of a chronic persistent cough. However, these children should experience short breaks in their symptoms in between infections. This is more likely in crowded homes where children are exposed to environmental tobacco smoke. Some viral infections cause prolonged periods of continuous cough ("post-infectious" or "post-viral" cough), and some viral infections or atypical bacteria can result in a "pertussis-like" coughing syndrome. In young infants, Chlamydia, cytomegalovirus, and Ureaplasma urealytica infections may also be involved.

In the "pertussis-like" illnesses the cough is often spasmodic and choking in nature, and may result in the classical whoop or in vomiting. Pertussis, parapertussis, adenovirus, mycoplasma and Chlamydia have all been implicated in causing this pattern of coughing. Serological tests, if positive, are reassuring. After the initial paroxysms, pertussis may remain symptomatic for many months and unresponsive to treatment.

The possibility of tuberculosis should also be considered. Tuberculosis may cause chronic cough, especially if there is secondary infection due to airways obstruction by protruding or perforating lymph nodes. In such cases the symptoms may temporarily respond to broad spectrum antibiotics. If airway infections are unusually frequent or severe, a number of underlying diseases may be considered including IgG subclass deficiency and IgA deficiency, or granulocyte dysfunction syndromes. If chronic airway infection is accompanied by failure to thrive, cystic fibrosis should be considered.

\section{Cough as a manifestation of asthma}

In 1981 and 1982 both Cloutier and Hannaway separately described selected groups of children whose main symptom was cough but in whom they considered asthma the diagnosis. ${ }^{14}{ }^{15}$ These children with chronic cough had responded well and rapidly to anti-asthma treatment (usually theophylline), but some had evidence of a prolonged expiratory phase on auscultation and others had exercise induced changes in lung function. Some of these children may have had "hidden asthma" - that is, they had true asthma but simply were never heard to wheeze by the parents or doctor and therefore appeared to have isolated chronic cough. It is difficult to define "cough variant asthma" (CVA) since asthma itself is not clearly defined. CVA is commonly diagnosed in a child with persistent isolated non-specific coughing who has one or more of: (1) airways hyperresponsiveness, (2) airways eosinophilic inflammation, (3) atopy (personal in the immediate family), or (4) whose cough responds rapidly to anti-asthma medication.

A 2 year follow up study of children with chronic or recurrent cough treated for asthma showed that 71 of 106 had subsequently been confirmed as having doctor diagnosed asthma, although this study was limited by an imprecise definition of asthma (a "recurrent respiratory condition") and lack of a placebo arm. ${ }^{16}$ More recent epidemiological studies have not confirmed this finding and suggest that children with isolated chronic cough are in many ways different from those with asthma and wheezing. Comparison of cough frequencies between children with known asthma and healthy controls of school age showed no differences between attacks, but more severe cough during bronchoconstriction. ${ }^{17}$ Night cough appeared to be unrelated to lung function and hyperresponsiveness in wheezing children of school age. ${ }^{11}$ Cough without wheeze had a more favourable prognosis than cough with recurrent wheeze in preschool children from the Tucson cohort, and tended to resolve before the age of 6 in most cases. ${ }^{7}$ This study suggested that chronic cough differs from asthma in several aspects and may therefore have a different pathophysiology. There is increasing evidence that, in some children with prolonged episodes of dry cough, there is a transient increase in cough receptor sensitivity. ${ }^{12}$

Invasive studies of the cellular pathology of the airways of children with chronic cough are rare. Fitch et al ${ }^{18}$ looked for the presence of asthmatic type airway inflammation in children undergoing elective surgery who had a history of coughing. After intubation for the surgical procedure a small volume of bronchoalveolar lavage (BAL) fluid was obtained. Only three $(10 \%)$ of 29 children studied had evidence of increased BAL fluid levels of eosinophils. This suggested that only a minority had true asthma if defined as airways eosinophilic inflammation. ${ }^{18}$ In another BAL study the inflammatory cell profile of children with chronic cough was more like that of controls than atopic asthmatics with a relative increase in neutrophils. ${ }^{19}$ In a community based study using induced sputum the symptom "wheeze" was a good discriminator for the presence of sputum eosinophilia (present in 45\%), whereas evidence of eosinophilic bronchitis was present in only $20 \%$ of the children with isolated cough. ${ }^{20}$ Zimmerman et al found that induced sputum from a group of children with "post-infectious cough" did not suggest airway eosinophilia compared with children with untreated allergic asthma, but they did observe that some of these children had hyperreactive airways. ${ }^{21}$

The presence of airways hyperresponsiveness was not associated with a response to anti-asthma treatment in children with persistent recurrent isolated cough. ${ }^{13}$ This suggests that the presence of bronchodilator responsiveness is not a very helpful tool for diagnosing CVA responsive to anti-asthma treatment. This is supported by a recent study which showed that, compared with atopy, a reduction in airways resistance after a bronchodilator was not a good discriminator between children with isolated persistent cough and those with wheezing. ${ }^{22}$

Clearly, the patterns described above have overlapping features. When combined, these studies suggest that most children with isolated non-specific cough do not have asthma as defined by eosinophilic airways inflammation. Thus, epidemiological studies and studies of bronchial inflammation do not support a strategy of "lumping" together all children with the diagnostic term "cough variant asthma", and to do so would lead to potential overdiagnosis of asthma. ${ }^{23}$

Several epidemiological studies have shown that chronic isolated and non-specific cough improves with time without treatment. $^{24-26}$

\section{PSYCHOGENIC COUGH}

Psychogenic cough can be defined as chronic dry cough without evidence of underlying illness. There is little 
published information on this type of cough, which may present as a habit "tic-like" cough (a dry repetitive and purposeless cough that persists after a simple upper respiratory infection) or as a bizarre "honking" cough (the child is indifferent to the cough but the parents are usually extremely worried about it). In both types of psychogenic cough the symptoms typically disappear at night during sleep. The cough abates when the child is engrossed in an activity but becomes more noticeable with attention. Characteristically, these children are older ( $>5$ years), are typically bright students, and are eager to please. Children with a "honking" psychogenic cough often suffer from other somatic symptoms and are taken from one specialist to another in search of a physical diagnosis. Family life and school attendance are disrupted and a vicious circle is established. Approach to treatment ranges from benign neglect (shifting the focus of attention to everyday concerns and away from the symptom) to hypnosis and family therapy. In many cases the cough is a manifestation of underlying stress and conflict. Although treatments such as wrapping the child in a tight fitting sheet may work dramatically on some occasions, such manoeuvres have an aversive effect and do not address the underlying conflict. ${ }^{27-29}$

\section{Reflux and aspiration}

Reflux with or without aspiration of gastric content or food is probably one of the most common paediatric causes of chronic respiratory symptoms including cough and wheeze. ${ }^{30}$ Both micro-aspiration and vagal reflexes elicited in the oesophagus may result in respiratory symptoms. Whether or not reflux and aspiration are a cause of chronic cough remains difficult to estimate on the basis of medical history. Some degree of gastro-oesophageal reflux is common in infants and improves with time. The presence of lipid laden macrophages in BAL fluid may reflect chronic aspiration, ${ }^{31}$ but it is also a feature of other chronic respiratory disorders and hence probably not very specific for aspiration as the primary cause of cough. ${ }^{32} 33$

\section{CHILDREN WITH CHRONIC COUGH IN WHOM THERE IS A SERIOUS UNDERLYING LUNG CONDITION}

Warning signs for serious underlying lung disease requiring further specialist investigations include:

- Neonatal onset of the cough: this suggests:

- a congenital defect that may be a problem with feeding and pulmonary aspiration;

- a problem with ciliary function and infection, including cystic fibrosis or primary cilial abnormality;

- an anatomical lesion in the airways (e.g. a cyst compressing airway or tracheomalacia); or

- a chronic viral pneumonia (e.g. CMV or Chlamydia) acquired in utero or during the perinatal period.

- Chronic moist or purulent cough: children $<5$ years usually swallow sputum and this may only be apparent when they vomit.

- If the cough started and persists after a choking episode: the choking episode of the inhaled foreign body may not always have been witnessed.

- If the cough occurs during or after feeding: this suggests gastro-oesophageal reflux or direct pulmonary aspiration while feeding.

- If there is general ill health with failure to thrive: finger clubbing or respiratory crackles on auscultation, lung infections that will not clear up, or other unresponsive infections elsewhere.
Chronic purulent cough suggests that there is chronic suppurative lung disease.

The following specific causes of chronic cough in childhood deserve consideration:

- chronic bronchitis;

- $\alpha_{1}$-antitrypsin deficiency;

- ciliary dyskinesia;

- congenital anatomical abnormalities;

- pulmonary aspiration.

\section{Chronic bronchitis}

These children often have a history of chronic cough with sputum production. Care must be taken to exclude other diagnoses such as cystic fibrosis, immune deficiency, or other conditions leading to bronchiectasis.. ${ }^{35}$ Some of these children respond well to a prolonged course of antibiotics (for example, 3-4 weeks of Augmentin) combined with physiotherapy.

\section{$\alpha_{1}$-antitrypsin deficiency}

$\alpha_{1}$-antitrypsin deficiency may occasionally present with problem coughing.

\section{Ciliary dyskinesia}

Congenital abnormalities of ciliary function impair mucociliary clearance of the airways and lead to chronic respiratory symptoms including cough. A diagnosis of primary ciliary dyskinesia should be suspected in children with a combination of unusually severe ear, nose and throat infections and lower airway infections. Situs inversus is present in about $50 \%$ of patients with primary ciliary dyskinesia and may be recognised prenatally on routine echography.

\section{Congenital anatomical abnormalities}

Anatomical abnormalities of the airways may cause chronic cough. Tracheobronchomalacia may occur as an isolated abnormality or as part of a syndrome-for example, VACTERL syndrome. Tracheo-oesophageal fistula (TOF) or laryngeal cleft causes cough due to aspiration, especially during meals. Any cause of airway compression or stenosis, including coarctation of the aorta and pulmonary sling and other vascular malformations, may cause chronic respiratory symptoms. These children have a "seal-like" barking cough which is particularly severe with concomitant viral upper respiratory tract infections. Tracheal causes include compressive lesions such as vascular rings, cysts, haemangiomas or mediastinal masses. Children who have been operated on at birth for a TOF or oesophageal atresia usually have tracheomalacia and a particularly disabling cough, often called the "TOF cough". With the passage of time (often years), the tracheal cartilages strengthen and the cough subsides. Sometimes a child with mild to moderate tracheal indentation from an aberrant right innominate artery can suffer problematic coughing, especially with head colds.

\section{Pulmonary aspiration}

This may be a retained foreign body or recurrent pulmonary aspiration during swallowing (if the swallow-breath sequence is compromised or poorly developed) or after swallowing (secondary to gastro-oesophageal reflux). Aspirated foreign bodies may go unrecognised for prolonged periods of time, especially if the diagnosis is missed initially. After an initial misdiagnosis of "pneumonia", a mean interval of 55 days until bronchoscopic extraction of the foreign body was reported by Hoeve et al. ${ }^{36}$ Foreign body aspiration is much more common in boys than in girls, and is especially frequent under the age of 4 years. The onset of symptoms is usually acute in previously symptom-free 
children. Godfrey et $a^{37}$ reported a $100 \%$ success rate for bronchoscopy in 26 children in whom foreign body aspiration was considered possible but by no means clear.

\section{COMPLICATIONS OF CHRONIC COUGH}

Coughing may have deleterious effects in addition to the beneficial effects of airway clearing. Extremely forceful coughing may induce bronchospasm, cause a rib fracture, an air leak (pneumomediastinum or pneumothorax), or syncope. More commonly, forceful coughing may be associated with a pulled intercostal or rectus abdominis muscle.

\section{DIAGNOSTIC APPROACH}

It is often difficult to arrive at a specific diagnosis and there is no easily performed diagnostic test in these children with isolated non-wheezing, non-specific coughing.

It is especially difficult to differentiate cough variant type asthma from non-asthmatic cough. In older children (>5-6 years) it may be possible to measure induced sputum for eosinophils, exhaled nitric oxide (increased in eosinophilic airways inflammation) or to do an exercise or methacholine challenge test. Helpful findings suggesting asthma related cough include:

- definite cough (in between head colds) to known asthma triggers;

- no evidence of alternative diagnosis (a normal or hyperinflated chest radiograph is also reassuring);

- correct setting-for example, other atopy or strong family history of asthma/atopy;

- clearcut response to a therapeutic trial of anti asthma medication. This may be a spontaneous improvement and therefore a relapse on stopping medication or when the "bad" season occurs again, and a second clearcut response to treatment is required before a diagnosis of asthma can be made..$^{38}$ One danger of such a trial is that, if it does not work, the anti-asthma medication is not stopped. Parents often report that the medication has worked a "bit". It is then tempting to think that an even bigger dose of medication might work a "bit better". This may turn out to be correct, but at some stage it is important to review the overall benefit of treatment. If the diagnosis of asthma is confirmed, then the lowest dose of inhaled steroid that maintains symptom control should be used.

Most children with non-specific coughing do not have asthma and do not respond well to bronchodilators or inhaled corticosteroids. Oral salbutamol is not an effective treatment for acute non-asthmatic coughing. ${ }^{39}$ Inhaled beclomethasone did not have a beneficial effect, ${ }^{13}{ }^{40}$ but Davies et al achieved mild to moderate benefit with a short 2 week course of high dose fluticasone ( $500 \mu \mathrm{g}$ twice daily). ${ }^{26}$

History taking should establish the severity and time course of the cough. Special features including diurnal variability, fever, colds, relation with meals and possible foreign body aspiration, habitual vomiting, production of sputum, risk of contact with tuberculosis or HIV, smoking behaviour of parents, possible allergies, and vaccination status, should be sought.

Relevant physical signs include ear, nose and throat infection, nasal polyps, chest deformities, abnormal chest auscultation, evidence of atopic disease including eczema, failure to thrive as evident from low weight for height and, sometimes, a low height for age. Deviation of the growth curve is a particularly important finding that indicates severe disease. It may be helpful to ask the child to perform his/her usual cough. This may help to differentiate between dry and productive cough, and reveal tracheobronchomalacia.
Additional tests include chest radiography and CT scanning, sputum cultures and serology, blood tests and screening for immunodeficiency, lung function tests, bronchoscopy, assessment of reflux, sweat test, and ciliary function studies to exclude primary ciliary dyskinesia.

Chest radiography may reveal congenital abnormalities, radio-opaque foreign bodies, infiltrates and atelectases, or show enlargement of the heart suggesting the presence of congenital heart disease (e.g. atrium septal defect). CT scanning is helpful in determining the presence of bronchiectasis which may not be evident on plain chest radiographs.

If there is any sputum, gram stains and microbiological cultures should be performed. Serological tests may be done to exclude infection with $B$ pertussis and relevant viruses. Determination of immunoglobulins and subclasses, and other tests of immunocompetence, are indicated in selected cases with chronic infections. Spirometric and bronchoprovocation tests may reveal reversible obstruction and hyperresponsiveness, or may suggest anatomical abnormalities including airway stenosis or malacia.

Bronchoscopy is indicated whenever there is any doubt of foreign body aspiration, in case of suspected congenital anomalies, or in order to obtain specimens from the lower airways for culture and microscopy. An increased number of lipid laden macrophages in the BAL fluid may reveal microaspiration.

Reflux studies are warranted when there is a suggestive medical history of reflux and should be considered in every infant with chronic cough. Barium swallow should be complemented by 24 hour oesophageal pH measurements as the golden standard for reflux assessment.

A sweat test to exclude cystic fibrosis should be done in children with chronic cough and failure to thrive, and in any child with chronic productive cough. It is mandatory that sweat tests are performed by experienced personnel according to established methodology (preferably according to Gibson and Cooke ${ }^{41}$ ).

Primary ciliary dyskinesia should be excluded by specialised centres, and this requires functional assessment in vivo or in vitro, electron microscopy, and epithelium cultures.

A diagnostic algorithm for children with chronic cough is shown in fig 1.

\section{TREATMENT OF CHRONIC COUGH IN CHILDREN}

The treatment of chronic cough in children should always be preceded by a systematic effort to exclude serious underlying illness and establish the cause of the cough.

Management of the otherwise well child with a persistent dry non-productive cough will include reassurance with watchful anticipation, since the cough will usually disappear in the next 4-8 weeks. Exposure to cigarette smoke should be removed whenever possible. Non-prescription cough remedies have not been shown to be efficacious in controlled trials for coughing with head colds, and cough suppressants may have side effects when given to young infants. ${ }^{41}$ Anti-asthma treatment is unlikely to be beneficial except in a minority who have true CVA. ${ }^{13-15} 3940$ If a "diagnostic trial" of antiasthma medication has failed to show benefit, it is important that it is discontinued. Several authors have anecdotally reported their successful experience of using nebulised lignocaine in refractory post-infectious dry cough in adults and children. ${ }^{42} 43$

Other therapeutic options include appropriate antibiotics for established or suspected infections, management of allergic airways disease with avoidance strategies and inhaled steroids, or surgical management of anatomical abnormalities. Reflux may be treated medically or surgically (Nissen fundoplication) in cases of severe oesophagitis unresponsive 


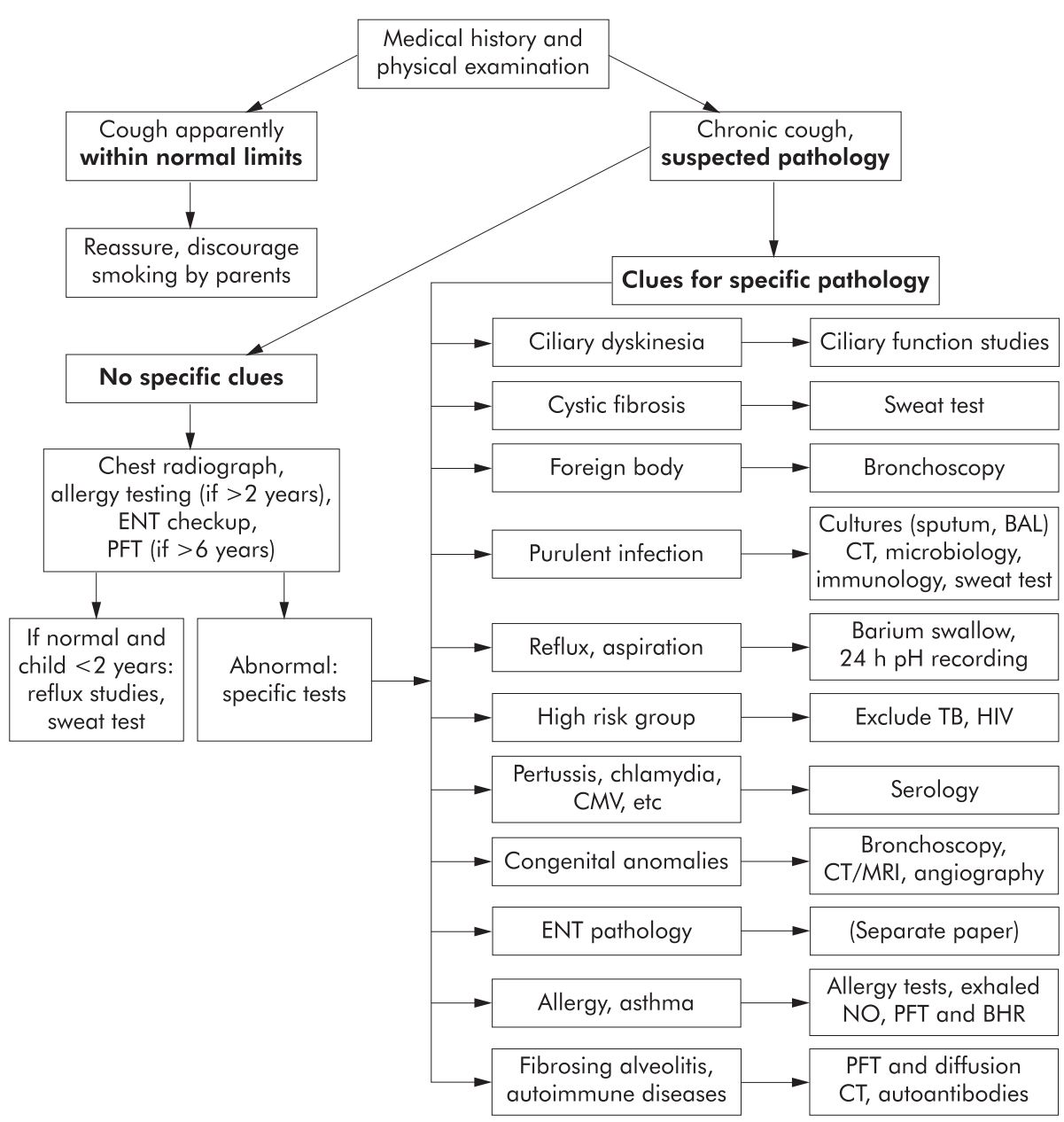

Figure 1 Diagnostic algorithm for use in children with chronic cough.

to conservative management. Cough will rarely be a reason for surgical intervention.

Antitussives are sometimes helpful in cases of otherwise untreatable cough, especially due to viral infections, but should not be given as first line treatment as they may retard proper diagnostic and therapeutic procedures. Codeine is probably the most effective central cough suppressor, but a variety of remedies are available which combine a potential pharmacological action with an important placebo effect.

There is no place for mucolytic agents including bromhexine or acetylcysteine as treatment for chronic cough, because there is no evidence that they work and because they may cause additional airway irritation. Human recombinant DNase may be effective in children in whom viscous purulent secretions are present and is indicated in cystic fibrosis.

\section{Authors' affiliations}

J C de Jongste, Department of Pediatric Respiratory Medicine, Sophia Children's Hospital/Erasmus MC University Medical Center, Rotterdam, The Netherlands

M D Shields, Department of Child Health, Queen's University of Belfast and The Royal Belfast Hospital for Sick Children, Belfast, UK

\section{REFERENCES}

1 Archer LNJ, Simpson H. Night cough counts and diary card scores in asthma. Arch Dis Child 1998;60:473-4.

2 Falconer A, Oldman C, Helms P. Poor agreement between reported and recorded nocturnal cough in asthma. Pediatr Pulmonol 1993;15:209-11.

3 Chang $A B$, Newman RG, Carlin JB, et al. Subjective scoring of cough in children: parent-completedv's child-completed diary cards vs an objective measure. Eur Respir J 1998;1 1:462-6.
4 Munyard P, Bush A. How much coughing is normal? Arch Dis Child 1996;74:531-4

5 Shahn F. How offen do children cough? Lancet 1996;384:699-700.

6 Faniran AO, Peat JK, Woolcock AJ. Measuring persistent cough in children in epidemiological studies: development of a questionnaire and assessment of prevalence in two countries. Chest 1999;115:434-9.

7 Wright AL, Holberg CJ, Morgan WJ, et al. Recurrent cough in childhood and its relation to asthma. Am J Respir Crit Care Med 1996;153:1259-65.

8 Kelly YJ, Brabin BJ, Milligan PJM, et al. Clinical significance of cough and wheeze in the diagnosis of asthma. Arch Dis Child 1996:75:489-93.

9 Charlton A. Children's coughs related to parental smoking. BMJ 1984;288: 1647-9.

10 Luyt DK, Burton PR, Simpson H. Epidemiological study of wheeze, doctor diagnosed asthma, and cough in preschool children in Leicestershire. BMJ 1993:306:1386-90.

11 Brooke AM, Lambert PC, Burton PR, et al. Recurrent cough: natural history and significance in infancy and early childhood. Pediatr Pulmonol 1998;26:256-61.

12 Chang AB, Phelan PD, Sawyer SM, et al. Airway hyperresponsiveness and cough-receptor sensitivity in children with recurrent cough. Am J Respir Crit Care Med 1997; 155:1935-9.

13 Chang AB, Phelan PD, Carlin J, et al. Randomised controlled trial of inhaled salbutamol and beclomethasone for recurrent cough. Arch Dis Child 1998;79:6-11.

14 Cloutier MM, Loughlin GM. Chronic cough in children: a manifestation of airway hyperactivity. Pediatrics 1981;67:6-11.

15 Hannaway $P$, Hooper D. Cough variant asthma in children. JAMA 1982;247:206-8.

16 Spellman R. Two-year follow up of the management of chronic or recurrent cough in children according to an asthma protocol. Br J Gen Pract 1991:41:406-9.

17 Rietveld S, Rijsenbeek-Nouwens LHM. Diagnostics of spontaneous cough in childhood asthma: results of continuous tracheal sound recording in the homes of children. Chest 1998;113:50-4.

18 Fitch PS, Brown V, Schock BC, et al. Chronic cough in children: bronchoalveolar lavage findings. Eur Respir J 2000;16:1109-14.

19 Marguet C, Jouen-Boedes F, Dean TP, et al. Bronchoalveolar cell profiles in children with asthma, infantile wheeze, chronic cough, or cystic fibrosis. Am J Respir Crit Care Med 1999;159:1533-40. 
20 Gibson PG Simpson لl, Chalmers AC, et al. Airway eosinophilia is associated with wheeze but is uncommon in children with persistent cough and frequent chest colds. Am J Respir Crit Care Med $2001 ; 164: 977-81$.

21 Zimmerman B, Silverman FS, Tarlo SM, et al. Induced sputum: comparison of postinfectious cough with allergic asthma in children. J Allergy Clin Immunol 2000;105:495-9.

22 McKenzie SA, Mylonopoulou M, Bridge PD. Bronchodilator responsiveness and atopy in 5-10 yr old coughers. Eur Respir J 2001;18:977-81.

23 McKenzie S. Cough - but is it asthma? Arch Dis Child 1994;70:1-2.

24 Brooke AM, Lambert PC, Burton PR, et al. Night cough in a population-based sample of children: characteristics, relation to symptoms and associations with measures of asthma severity. Eur Respir J 1996;9:65-71.

25 Powell CVE, Primhak RA. Stability of respiratory symptoms in unlabelled wheezy illness and nocturnal cough. Arch Dis Child 1996:75:549-54.

26 Davies MJ, Fuller P, Picciotto A, et al. Persistent nocturnal cough: randomised controlled trial of high dose inhaled corticosteroids. Arch Dis Child 1999;81:38-44.

27 Lavigne JV, Davis T, Fauber R. Behavioural management of psychogenic cough: alternative to the bedsheet and other aversive techniques. Pediatrics 1991;87:532-7.

28 Milner AD. Psychogenic cough in childhood. BMJ 1985;290:1847-8.

29 Gay MG, Blager F, Bartsch K, et al. Psychogenic habit cough: review and case reports. J Clin Psychiatry 1987;48:483-6.

30 Harding SM, Richter JE. The role of gastroesophageal reflux in chronic cough and asthma. Chest 1997;111:1389-402.

31 Colombo JL, Hallberg TK. Pulmonary aspiration and lipid-laden macrophages: in seqarch of gold (standards). Pediatr Pulmonol 1999;28:79-82.
32 Knaver-Fischer S, Ratjen F. Lipid-laden macrophages in bronchoalveolar lavage fluid as a marker for pulmonary aspiration. Pediatr Pulmonol 1999;27:419-22.

33 Kazachkov MY, Muhlebach MS, Livasy CA, et al. Lipid-laden macrophage index and inflammation in bronchoalveolar lavage fluids in children. Eur Respir J 2001;18:790-5.

34 Seear M, Wensley D. Chronic cough and wheeze in children: do they all have asthma? Eur Respir J 1997; 10:342-5.

35 Peat JK, Woolcock AJ, Leeder SR. Asthma and bronchitis in Sydney children. I. Prevalence during a six year study. Am J Epidemiol 1980;111:721-7.

36 Hoeve LJ, Rombout J, Pot DJ. Foreign body aspiration in children. The diagnostic value of signs, symptoms and pre-operative examination. Clin Otolaryngol 1993; 18:55-7.

37 Godfrey S, Avital A, Maayan C, et al. Yield from flexible bronchoscopy in children. Pediatr Pulmonol 1997;23:261-9.

38 Bush A. Chronic cough and/or wheezing in infants and children less than 5 years old: diagnostic approaches. In: Naspitz CK, Szefler SJ, Tinkelman DG, Warner JO, eds. Textbook of pediatric asthma - an international perspective. London: Martin Dunitz, 2001.

39 Bernard DW, Goepp JG, Duggan AK, et al. Is oral albeterol effective for acute cough in non-asthmatic children. Acta Paediatr 1999;88:465-7.

40 Evald T, Munch EP, Kok-Jensen A. Chronic non-asthmatic cough is not affected by inhaled beclomethasone dipropionate. A controlled double blind clinical trial. Allergy 1989;44:510-4.

41 Smith MB, Feldman W. Over-the-counter cold medications. A critical review of clinical trials between 1950 and 1991. JAMA 1993;269:2258-63.

42 Almansa-Pastor A. Treating refractory cough with aerosols of mepivacaine. Chest 1996:110:1374-5.

43 Trochtenburg S. Nebulised lidocaine in the treatment of refractory cough. Chest 1994;105:1592-3.

LUNG ALERT

Nodule size on HRCT scanning may predict infectious agent aetiology in immunocompromised patients with suspected pulmonary infection

$\Delta$ Franquet T, Muller NL, Gimenez A, et al. Infectious pulmonary nodules in immunocompromised patients: usefulness of computed tomography in predicting their etiology. J Comput Assist Tomogr 200;27:461-8

$\mathrm{T}$ his review of HRCT findings in 78 immunocompromised patients with a diagnosis of a proven infectious agent included patients with AIDS $(n=25)$ or bone marrow

transplantation $(n=21)$. Patients with typical HRCT features of infectious bronchiolitis and endobronchial spread of tuberculosis (branching linear or nodular opacities, tree-in-bud pattern) were excluded.

Nodules were categorised by size, cross sectional distribution, and five other features. $92 \%$ had multiple bilateral nodules. Infectious agents were Mycobacterium $(\mathrm{n}=24)$, fungi $(\mathrm{n}=22)$, and viruses $(\mathrm{n}=12)$. There was no case of miliary tuberculosis. Univariate analysis showed that absence of cavitation, small size, and "halo sign" were significantly associated with viral aetiology. Multivariate analysis indicated that a diameter of $<10 \mathrm{~mm}$ was the only independent predictor of aetiology $(\mathrm{p}<0.0001)$.

The authors conclude that, in immunocompromised patients with multiple non-miliary nodules on CT scanning and suspected pulmonary infection, nodule size of uniformly $<10 \mathrm{~mm}$ predicts a viral aetiology.

C M O'Driscoll

Consultant Radiologist, Royal United Hospital, Bath, UK Martin.ODriscoll@ruh-bath.swest.nhs.uk 